\title{
Producción y rendimiento de maíz en cuatro tipos de labranza bajo condiciones de temporal
}

\author{
Production and yield of corn in four types of farming \\ in conditions of temporary \\ José Luis Olguín López¹, Rubén Dario Guevara Gutiérrez1*, Juan Arturo Carranza Montaño², \\ Eric Scopel, Oscar Arturo Barreto García ${ }^{1}$, Oscar Raúl Mancilla Villa ${ }^{1}$ y Antonio Talavera Villareal ${ }^{1}$
}

\section{RESUMEN}

El aumento en la demanda de los alimentos ha obligado al hombre a la búsqueda y adopción de sistemas y técnicas de producción. En el presente trabajo se cuantificó el rendimiento en el cultivo de maíz en cuatro sistemas de labranza: labranza de conservación (Lc), labranza cero sin mantillo (Lo), labranza superficial con rastra ( $\mathrm{Rm}$ ) y labranza con arado de discos (Ar); considerando factores como densidad de plantas, mazorcas por planta, y granos por mazorca. El diseño experimental utilizado fue parcelas divididas con dos factores y dos repeticiones por localidad; el factor B representado por la parcela chica bajo los cuatro tratamientos; como parcela dividida en el factor A, representada por la parcela grande aplicando subsoleo y sin subsoleo. La ubicación del estudio fue en las parcelas localidades: La Tinaja, La Croix, San Isidro y Alista; la semilla empleada fue criolla de la zona, empleándose $20 \mathrm{~kg}$ $\mathrm{ha}^{-1}$, la fertilización empleada fue la fórmula 100 - 60 - 00 depositándose a la siembra todo el fósforo, el restante distribuido a la primera y segunda escarda. Los resultados obtenidos manifiestan que el rendimiento del maíz fue menor en labranza cero sin mantillo, los mejores resultados fueron para Lc con 8 ton ha $\mathrm{h}^{-1}$ las comunidades de la Tinaja y la Croix; para Ar se registraron valores superiores a 6 ton ha ${ }^{-1}$ en los sitios de San Isidro y Alista. De la comparación entre tratamientos se determina que las condiciones de clima y suelo ejercen variabilidad sobre el rendimiento, identificando que la Lc no siempre resulta la mejor opción para obtener los mejores rendimientos ya que Ar también alcanzó valores superiores en otros sitios.

Palabras clave: agricultura, clima, tratamientos, suelo.

\begin{abstract}
The increase in demand for food has forced human beings to the search and adoption of production systems and techniques. In this paper, the maize yield was quantified in four tillage systems: conservation tillage (Lc), no-tillage without mulch (Lo), shallow tillage with harrow (Rm) and disc plow tillage (Ar); considering factors such as plant density, ears per plant and grains per ear. The experimental design was split plots with two factors and two replications per locality; $B$ factor represented by the small plot under four treatments; split plot in the A factor, represented by the large plot using subsoiling and without subsoiling. The location of the study was on plots in the locations: La Tinaja, La Croix, San Isidro and Alista; Creole seed was used in the area, using $20 \mathrm{~kg} \mathrm{ha}^{-1}$, the used fertilization was the 100-60-00 Formula; all Phosphorus was deposited on the sowing, the remaining was distributed at the first and second weeding. The obtained results demonstrated that the maize yield was lower in no-tillage without mulch treatment, the best results were at Lc with 8 ton ha ${ }^{-1}$ in "La Tinaja" and "La Croix" communities; For Ar, higher than 6 ton $\mathrm{ha}^{-1}$ values were registered in the San Isidro and Alista sites. From the comparison among treatments, it is determined that the conditions of climate and soil exert variability on yield, identifying that Lc is not always the best option to get the best yield since Ar also reached higher values in other sites.
\end{abstract}

Key words: agriculture, climate, treatment, soil.

1 Centro Universitario de la Costa Sur, Universidad de Guadalajara. Av. Independencia Nacional 151. Autlán de Navarro, Jalisco, México.

2 Secretaría del Medio Ambiente y Desarrollo Territorial (SEMADET). Álvaro Obregón 7. Autlán de Navarro, Jalisco, México.

* Autor por correspondencia: rguevara@cusur.udg.mx, rubendariogg77@gmail.com

Fecha de Recepción: 5 Abril, 2016.

Fecha de Aceptación: 19 Febrero, 2016.

DOI: $10.4067 /$ S0718-34292017005000018 


\section{Introducción}

La actividad agrícola en México es caracterizada por la baja constante de los precios de su producción, ejemplo de ello es el cultivo de maíz, el más utilizado para consumo humano y animal (OCDE/FAO, 2013). García y Rodríguez (2012) establecen que en México la superficie sembrada de maíz en condiciones de temporal es de 15,5 millones de hectáreas, $75 \%$ de la superficie total agrícola de temporal. Gútierrez et al. (2006) identifican que a nivel mundial este cultivo ocupa el cuarto lugar en superficie sembrada y en producción. INEGI (2013) reconoce que este cultivo representa en México 50,3\% de la superficie agrícola cultivada. Wang et al. (2014) identifican que la producción de cultivos como el maíz es importante por su consumo excesivo, su alto valor nutritivo, económico y médico. Bauer y Black (1994) y Cao et al. (2011) manifiestan el efecto favorable de la combinación maíz-frijol en la producción de nutrientes y en la mejora física, química y biológica del suelo, haciendo de ellos buenos indicadores de la calidad y productividad. El maíz intercalado con leguminosas alimentarias mencionan Buckles y Barreto (1996) se considera un factor de éxito de uso generalizado en la producción de alimentos.

Bergamaschi et al. (2006), mencionan que el cultivo del maíz está firmemente influenciado por el comportamiento de la precipitación y el déficit hídrico que causa mayor impacto sobre el rendimiento del grano cuando ocurre la floración. Para determinar su influencia se estableció un balance hídrico del suelo a $20 \mathrm{~cm}$ de profundidad cuyos resultados manifiestan que el déficit hídrico durante la floración repercute sobre el rendimiento. Otros componentes que influyen en el rendimiento y producción de maíz son los abonos orgánicos, por aportar nutrientes y microorganismos que favorecen a la fertilidad del suelo y nutrición de las plantas como lo manifiesta Eghball et al. (2004), que mencionan el efecto de los abonos orgánicos en el rendimiento, los que pueden variar de 2.152 a $3.616 \mathrm{~kg}$ grano ha-1, respondiendo el valor más alto a la aplicación del humus de lombriz.

En contexto, los sistemas de labranza ejercen efectos diferenciales en el rendimiento de los cultivos, en las características físicas, químicas e hidráulicas de los suelos. Bajo el sistema de labranza convencional, el uso del arado y rastra modifica la estructura de la capa superficial del suelo, la continuidad del espacio poroso y reduce el contenido de materia orgánica (Paustian et al., 1997). Los residuos de cosecha se mezclan y se exponen a los ciclos acelerados de aireación, humedecimiento, secado y enfriamiento, que sumados favorecen la descomposición de la materia orgánica, guardando estrecha relación con la porosidad y humedad; componentes que influyen directamente en la recuperación de la estructura y estabilidad cuando los suelos se exponen a diferentes tipos de manejo (Báez y Aguirre, 2011).

En los estudios de Ureste y Campos (1995), se manifiesta la respuesta del cultivo de maíz a la labranza de conservación en cuatro diferentes suelos tropicales bajo condiciones de temporal en comparación a los sistemas de labranza convencional reducida y labranza cero. Las variables medidas fueron densidad de población y rendimiento de grano, en suelo Fluvisol de textura fina y de textura media se obtuvo la mayor y más uniforme densidad de población, así como el mayor rendimiento de grano, no existiendo prácticamente diferencia entre estos dos suelos y entre los cuatro sistemas de labranza. En el suelo Luvisol de textura gruesa muy drenado, la densidad de población fue mayor en cero labranza que en la labranza reducida y convencional. Sin embargo, el rendimiento de grano obtenido con labranza reducida y cero fue prácticamente igual entre sí, pero superior al obtenido con la labranza convencional. En el suelo Vertisol arcilloso mal drenado se obtuvo el menor rendimiento de grano con los cuatro sistemas de labranza y este fue mayor en labranza convencional que en la labranza cero y reducida. En el suelo Vertisol arcilloso mal drenado no es posible producir maíz económicamente con cualquiera de los sistemas de labranza estudiados, a menos que se utilice alguna práctica para reducir el exceso de humedad del suelo.

En los estudios realizados por Galeana et al. (2000), se manifiesta que el rendimiento en tres ciclos de cultivo de maíz (Zea mays L.) fue superior bajo el sistema de labranza de conservación en comparación al sistema de labranza tradicional, obteniendo rendimientos de 3,19 ton ha ${ }^{-1}$ y 2,51 ton $\mathrm{ha}^{-1}$ respectivamente; respuesta que fue favorecida por la incorporación de cobertura de veza, adicional a ello se observó el abatimiento del $\mathrm{pH}$, el aumento de la materia orgánica y población de lombrices.

Para Ortiz-Torres et al. (2013), los rendimientos entre las poblaciones nativas de maíz cultivadas en el Valle de Tehuacán-Puebla, varían con base a 
las fuentes aplicadas de fertilización, obteniendo los mayores rendimientos en variedades locales con $11.595 \mathrm{~kg} \mathrm{ha}^{-1}$. Broa et al. (2013), reporta rendimientos de grano en maíces nativos de color a partir de la eficiencia de aplicación y el índice de productividad del agua, registrando valores de $2.039 \mathrm{~kg} \mathrm{ha}^{-1}$ y $4.163 \mathrm{~kg} \mathrm{ha}^{-1}$ como mínimo y máximo.

Díaz et al. (2014), mencionan que para zonas semiáridas el rendimiento óptimo del maíz está sustentado principalmente en la aplicación de agua y fertilización nitrogenada; las dosis de $\mathrm{N}$ consideradas fueron: óptima $477 \mathrm{~kg}$ de $\mathrm{N}^{\text {ha }}{ }^{-1}$ produciendo un rendimiento máximo de $9,89 \mathrm{Mg} \mathrm{ha}^{-1}$; y óptima económica $341 \mathrm{~kg}$ de $\mathrm{N} \mathrm{ha}^{-1}$ generando un rendimiento de $9,44 \mathrm{Mg} \mathrm{ha}^{-1}$.

Ante lo expuesto en el presente estudio se planteó conocer el rendimiento de maíz en zonas de temporal en el municipio de Venustiano Carranza, Jalisco-México, utilizando tratamientos de labranza bajo un diseño en parcelas divididas con dos factores (A y B). Los resultados generados están fundamentados en la efectividad en términos de producción bajo el sistema de labranza de conservación en comparación a sistemas tradicionales de labranza en zonas de pluviometría irregular. El régimen pluviométrico de la zona es inferior a $500 \mathrm{~mm}$.

\section{Materiales y Métodos}

El presente trabajo se estableció en el municipio de Venustiano Carranza, ubicado al sur del estado de Jalisco-México entre los 19³7'21.4" a $19^{\circ} 53^{\prime} 28.39^{\prime \prime}$ 'Norte y $103^{\circ} 39^{\prime} 45.52^{\prime \prime}$ ' a $104^{\circ} 02^{\prime} 46.71^{\prime \prime}$ Oeste. Esta zona es considerada como zona de pie de monte, ubicada en las faldas de los volcanes de Colima, con un rango altitudinal de 1.000 a 1.500 msnm (Figura 1). La precipitación pluvial media anual es de 669,23 mm (SMN-CNA, 2015); los suelos son color negro característicos de zonas semiáridas, profundidad superior a $80 \mathrm{~cm}$, su capa superior es rica en materia orgánica y nutrientes aunque se encuentra caliche disuelto o cementado en el subsuelo.

La unidad de monitoreo seleccionada fue establecida en cuatro parcelas experimentales con superficie de $1.000 \mathrm{~m}^{2}$ cada una de ellas, distribuidas en cuatro comunidades bajo un mismo diseño (Figura 2). Con base a la pluviometría irregular, estas fueron: Zona 1 o Seca, conformadas por las parcelas ubicadas en los ejidos La Tinaja (suelos pardos) y La Croix (suelos negros), de $\mathrm{pH} 7$ a 8, con $35 \%$ de arcilla, 25\% limo, $40 \%$ arena, y; Zona 2 o Húmeda, los ejidos de San Isidro (suelos pardos) y Alista (suelos negros), de pH 5 a 6, y

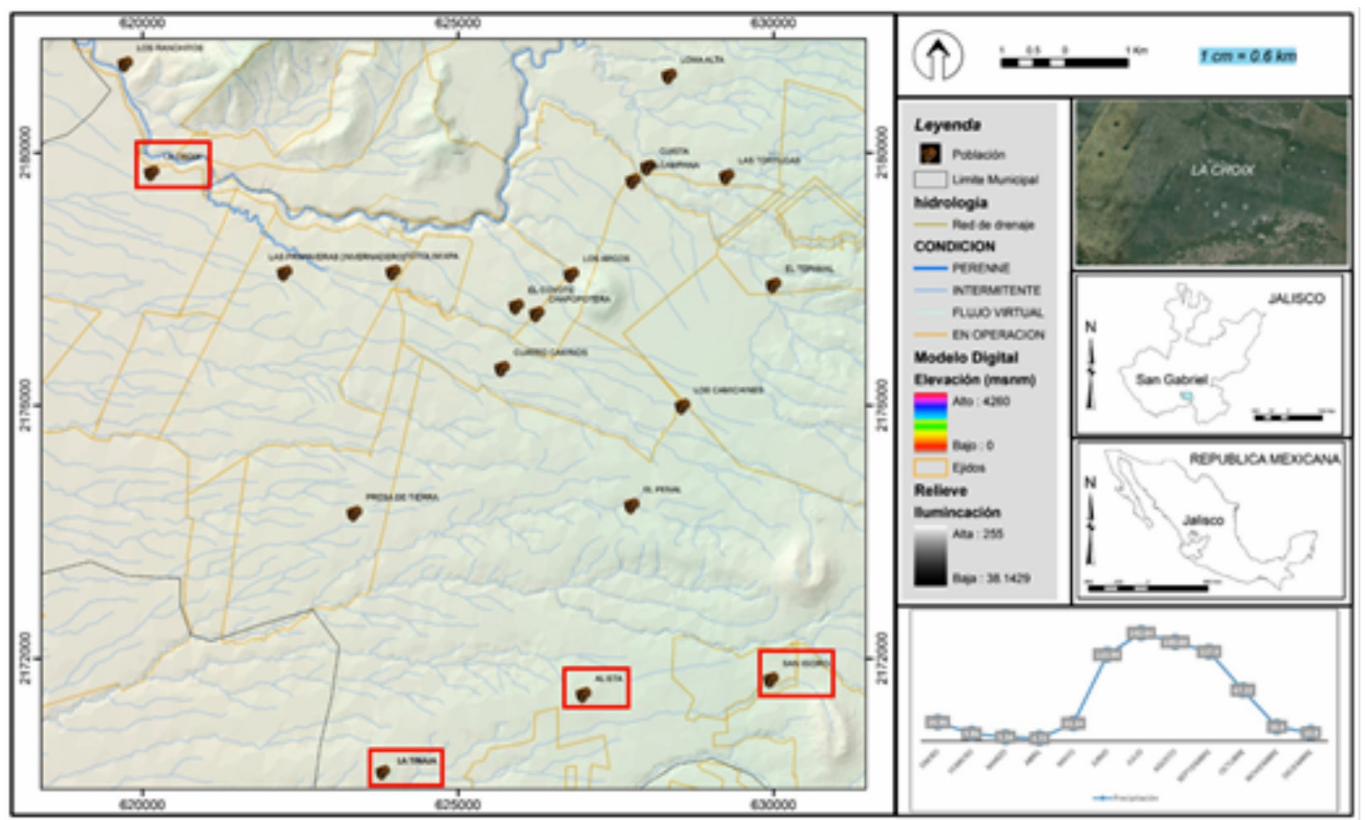

Figura 1. Localización de la zona de estudio. 
La Tinaja Alista San Isidro

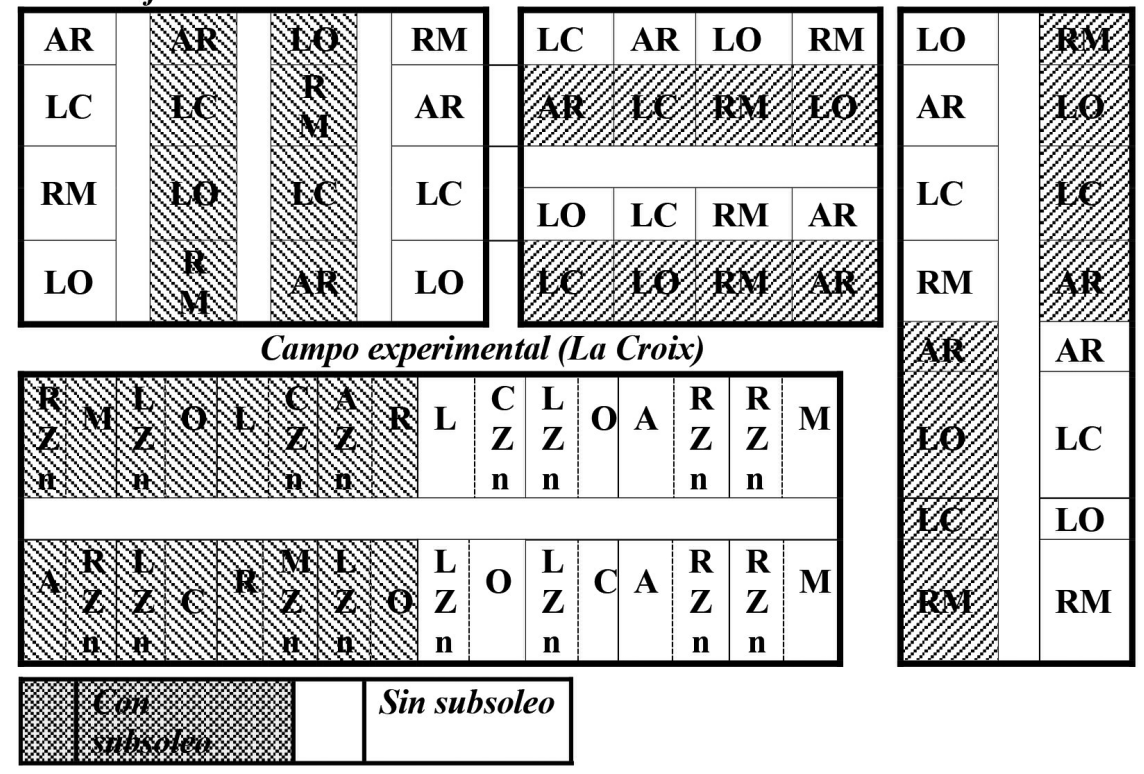

Figura 2. Diseño experimental con cuatro repeticiones.

textura con $10 \%$ arcilla, $25 \%$ limo, $65 \%$ arena. Las parcelas seleccionadas están caracterizadas por estar sembradas históricamente con maíz, por la presencia de sequias prolongadas que perjudican la condición óptima de agua disponible y por las condiciones edafológicas variadas.

El diseño experimental utilizado fue parcelas divididas, fundamentado en determinar las diferencias entre tratamientos y la magnitud entre estas (Badii et al., 2007). Bajo este contexto se considera verificar la tendencia derivada del análisis de los datos del experimento.

El diseño propuesto considera dos factores y dos repeticiones por localidad (Figura 2). El factor A, parcelas experimentales sin y con subsoleo de cincel (práctica mecánica) a una profundidad aproximada de $40 \mathrm{~cm}$, arado a $20 \mathrm{~cm}$ y distancia entre surcos de $75 \mathrm{~cm}$ realizados con tracción animal, ubicados de norte a sur (práctica en seco). El factor B, está conformado por cuatro subparcelas de $250 \mathrm{~m}^{2}$ para cada parcela, con separación entre ellas de 1,0 metro; los tratamientos considerados para estas fue: Labranza cero sin mantillo (Lo); Labranza de conservación (Lc) conformada por labranza cero más residuos vegetales $\left(3\right.$ ton ha $\left.\mathrm{h}^{-1}\right)$; Labranza superficial (8 a $15 \mathrm{~cm}$ ) con rastra $(\mathrm{Rm})$ más el control mecánico de malezas (escarda) y; Arado con discos (Ar), con paso de rastra y control mecánico de malezas.
Para la siembra se utilizó semilla criolla (amarillo) originaria de la zona, $20 \mathrm{~kg} \mathrm{ha}^{-1} \mathrm{y}$ densidad de 50.000 plantas, esta actividad se realizó con método de tapa-pie colocando dos semillas por golpe. La fertilización fue realizada al momento de la siembra con la fórmula 20-60-00 (fosfato de amonio) mezclada con $12 \mathrm{~kg}$ de Sulfato de zinc y aplicada en tres tratamientos a excepción de La Croix (campo experimental); la segunda fertilización fue al momento de la escarda con la fórmula 40-00-00 (nitrato de amonio).

En consideración a la descripción de las mediciones se planteó la utilización de los procesos metodológicos siguientes: a) Bajo el objetivo de estimar la materia seca producida (indicador del funcionamiento de la planta), se procedió a medir la altura de plantas (basal o $10 \mathrm{~cm}$ ) y diámetro, realizado en dos ocasiones (cinco semanas después de la siembra y al momento de la floración de las plantas). b) Para cada fecha establecida en el punto anterior, se calibró la relación materia seca y altura por diámetro al cuadrado sobre 60 plantas para verificar la correlación existente. Finalmente, c) Estimación del área foliar, a partir del que se determina la intensidad de fotosíntesis e intercambio de gases y agua, a las cinco semanas se delimitó un área de $2 \mathrm{~m}^{2}$ en donde se midió largo y ancho de lámina foliar en las hojas de todas las plantas, obteniéndose las diferencias de disponibilidad de agua entre tratamientos. 
La estimación del rendimiento fue realizado por ser considerado el mejor indicador de éxito a conveniencia de implementar esta técnica; esta se realizó de tres maneras: 1) En la parcela de San Isidro se delimitaron parcelas de $7 \operatorname{surcos} x 7 \mathrm{~m}$, cosechando lo que se encontró dentro de los límites; para saber el total del terreno cosechado, se realizó una corrección tomando en cuenta la distancia del intersurco. 2) Para la parcela de La Croix, se delimitaron 5 surcos por $5 \mathrm{~m}$, cosechándose únicamente los tratamientos en los que se agregó el zinc (en siembra) y, 3) En la parcela de La Tinaja y Alista la parcela útil se delimitó sobre 4 surcos por $2 \mathrm{~m}$ realizándose la cosecha de esta manera por tener problemas de acame.

Para cada uno de los sitios, en campo fueron seleccionadas al azar 10 mazorcas, las que fueron pesadas, marcadas con información de cada tratamiento y almacenadas. De esta información se obtuvieron las medidas de humedad, granos por mazorca y peso de $1.000 \mathrm{~g}$; con esta base, se analizó el comportamiento de los componentes de rendimiento que permiten entender de manera fisiológica las limitaciones al rendimiento.

\section{Resultados y Discusión}

La comparación entre sitios y tratamientos, en la localidad de Alista se registraron los mejores rendimientos, contrariamente La Tinaja que tiene el suelo más fértil (suelo Feozem) presentó rendimientos menores a 50\% respecto a los demás tratamientos (Tabla 1). En referencia a los registros de rendimiento con práctica sin subsoleo, en La Tinaja y La Croix se obtuvieron marcadas diferencias del tratamiento Lc respecto a otros tratamientos; para San Isidro y Alista los mejores registros de rendimiento fueron en el tratamiento Ar.

En la práctica sin subsoleo, el análisis de varianza del rendimiento por sitio (Tabla 2), se enmarca el valor de $\mathrm{F}<\mathrm{F}$, respuesta que manifiesta que las condiciones de suelo, prácticas aplicadas y clima no modifican la respuesta del cultivo.

El análisis de varianza entre sitios $\alpha=0,05$ muestra variabilidad marcada $\left(F>F^{\prime}\right)$ registrando resultados que demuestran que las condiciones de suelo, las prácticas aplicadas y el clima de la región influyen sobre el rendimiento (Tabla 3).

Tabla 1. Rendimiento total en $\mathrm{Kg} \mathrm{ha}^{-1}$ con tratamiento sin subsoleo.

\begin{tabular}{lcccccc}
\hline Sitios/Tratamientos & Lc & Lo & Rm & Ar & Factor B (\%)* & Clasificación \\
\hline La Tinaja & 4.870 & 920 & 2.964 & 2.250 & $\mathrm{AS}<5 \%$ & $\mathrm{Lc}>\mathrm{Ar}-\mathrm{Rm}>\mathrm{Lo}$ \\
La Croix & 7.220 & 3.650 & 4.185 & 5.014 & $\mathrm{AS}<5 \%$ & $\mathrm{Lc}>\mathrm{Ar}-\mathrm{Rm}>\mathrm{Lo}$ \\
San Isidro & 5.665 & 5.394 & 5.515 & 6.370 & $\mathrm{~S}<5 \%$ & $\mathrm{Ar}-\mathrm{Lc}-\mathrm{Rm}-\mathrm{Lo}$ \\
Alista & 5.910 & 5.244 & 5.825 & 8.525 & $\mathrm{AS}<5 \%$ & $\mathrm{Ar}>\mathrm{Lc}-\mathrm{Rm}-\mathrm{Lo}$ \\
\hline
\end{tabular}

$\mathrm{S}=$ Significativo, AS = Altamente significativo.

Tabla 2. Análisis de varianza con niveles de significancia de 0,01 sin subsoleo.

\begin{tabular}{lcccccc}
\hline \multicolumn{1}{c}{ Sitios } & Suma & Promedio & Varianza & F & Probabilidad & Valor crítico F' \\
\hline La Tinaja & 11,00 & 2,75 & 2,71 & 5.32 & 0.01 & 5,95 \\
Campo experimental & 20,07 & 5,02 & 2,47 & & & \\
San Isidro & 22,94 & 5,74 & 0,19 & & & \\
Alista & 25,50 & 6,38 & 2,14 & & & \\
\hline
\end{tabular}

Tabla 3. Análisis de varianza con $\alpha=0,05$ sin subsoleo.

\begin{tabular}{lcccccc}
\hline \multicolumn{1}{c}{ Sitio } & Suma & Promedio & Varianza & F & Probabilidad & Valor crítico F' \\
\hline La Tinaja & 11,00 & 2,75 & 2,71 & 5,32 & 0,01 & 3,49 \\
La Croix & 20,07 & 5,02 & 2,47 & & & \\
San Isidro & 22,94 & 5,74 & 0,19 & & & \\
Alista & 25,50 & 6,38 & 2,14 & & & \\
\hline
\end{tabular}


En práctica con subsoleo, el mayor rendimiento fue registrado en Alista con un total de 26,02 toneladas y el mínimo en La Tinaja con 12,48 toneladas, la diferencia es de 52\% (Tabla 4).

La variación del rendimiento total por sitio fue significativa, su comportamiento fue similar en San Isidro, La Croix y Alista, pero difieren entre tratamientos. En todos los tratamientos de Alista y San Isidro rebasan las 5 ton ha ${ }^{-1}$, comportamiento similar en la Croix y La Tinaja para Lo que justo alcanzó esta cifra.

El análisis de varianza con $\alpha=0,01$ presenta ligera variación en los promedios (Tabla 5), lo que sugiere que el rendimiento entre sitios no presenta variación; respuesta que manifiesta independencia con respecto a la práctica de subsoleo, tipo de suelo, condiciones de temporal y efecto de tratamientos.

La fuerte relación existente entre las condiciones de manejo de cada sitio considerando $\alpha=0,05$ (Tabla 6) se identifica en el rendimiento obtenido una relación que muestra la susceptibilidad del rendimiento a las condiciones climáticas entre sitios $\left(\mathrm{F}>\mathrm{F}^{\prime}\right)$.
El mayor registro de rendimiento total por tratamiento sin subsoleo fue para Lc (Tabla 7 y 8 ) no obstante los resultados del análisis de varianza $(\alpha=$ 0,01 y 0,05 ) manifiestan diferencias significativas, reconociéndose que Lc es superada en rendimiento por otros tratamientos; lo que se traduce que Lc no necesariamente obtendrá los mejores rendimientos en respuesta a la fertilización, características de suelo o clima $\left(\mathrm{F}<\mathrm{F}^{\prime}\right)$. La variación en rendimientos entre tratamientos muestra valores muy parecidos entre Lc y Ar, con respecto a Rm y Lo.

El rendimiento registrado en los tratamientos con subsoleo y sin subsoleo, resultaron ser muy semejantes, lo que hace suponer que esta práctica no modifica la respuesta entre tratamientos, no obstante se observa ligero aumento en los valores. Del análisis de varianza $(\alpha=0,01$ y 0,05$)$ para los tratamientos con subsoleo (Tablas 9 y 10) registraron una $\mathrm{F}<\mathrm{F}$ ' lo que se traduce que en $\mathrm{Lc}$ no siempre se obtendrán los mayores rendimientos, para el estudio $\mathrm{Rm}>\mathrm{Ar}>\mathrm{Lc}$ en los sitios de San Isidro y Alista.

Tabla 4. Rendimiento total en $\mathrm{Kg} \mathrm{ha}^{-1}$ por tratamiento con subsoleo.

\begin{tabular}{lcccccc}
\hline Sitios/Tratamiento & Lc & Lo & $\mathrm{Rm}$ & $\mathrm{Ar}$ & Factor B (\%)* & Clasificación \\
\hline La Tinaja & 5,000 & 1,500 & 3,000 & 2,980 & $\mathrm{AS}<5 \%$ & $\mathrm{Lc}>\mathrm{Ar}-\mathrm{Rm}>\mathrm{Lo}$ \\
Campo experimental & 7,900 & 3,900 & 4,300 & 4,500 & $\mathrm{AS}<5 \%$ & $\mathrm{Lc}>\mathrm{Ar}-\mathrm{Rm}>\mathrm{Lo}$ \\
San Isidro & 5,460 & 6,000 & 5,600 & 6,490 & $\mathrm{~S}<5 \%$ & $\mathrm{Ar}-\mathrm{Lc}-\mathrm{Rm}-\mathrm{Lo}$ \\
Alista & 5,700 & 5,860 & 6,200 & 8,260 & $\mathrm{AS}<5 \%$ & $\mathrm{Ar}>\mathrm{Lc}-\mathrm{Rm}-\mathrm{Lo}$ \\
\hline
\end{tabular}

$\mathrm{S}=$ Significativo, $\mathrm{AS}=$ Altamente significativos.

Tabla 5. Análisis de varianza con $\alpha=0,01$ con práctica de subsoleo.

\begin{tabular}{lcccccc}
\hline \multicolumn{1}{c}{ Grupos } & Suma & Promedio & Varianza & F & Probabilidad & Valor crítico F' \\
\hline La Tinaja & 12,48 & 3,12 & 2,06 & 4,87 & 0,02 & 5,95 \\
Campo experimental & 20,60 & 5,15 & 3,42 & & & \\
San Isidro & 23,55 & 5,89 & 0,21 & & & \\
Alista & 26,02 & 6,51 & 1,41 & & & \\
\hline
\end{tabular}

Tabla 6. Análisis de varianza con $\alpha=0,05$ con práctica de subsoleo.

\begin{tabular}{lllcccc}
\hline \multicolumn{1}{c}{ Sitios } & Suma & Promedio & Varianza & F & Probabilidad & Valor crítico F' $^{\prime}$ \\
\hline La Tinaja & 12,48 & 3,12 & 2,06 & 4,87 & 0,019 & 3,49 \\
Campo experimental & 20,6 & 5,15 & 3,42 & & & \\
San Isidro & 23,55 & 5,8875 & 0,21 & & & \\
Alista & 26,02 & 6,505 & 1,41 & & & \\
\hline
\end{tabular}


Tabla 7. Análisis de varianza con $\alpha=0,01$ sin subsoleo.

\begin{tabular}{ccccccc}
\hline Tratamiento & Suma & Promedio & Varianza & F & Probabilidad & Valor crítico F' \\
\hline Lc & 23,67 & 5,92 & 0,95 & 1,04 & 0,41 & 5,95 \\
Lo & 15,21 & 3,80 & 4,31 & & & \\
Rm & 18,49 & 4,62 & 1,73 & & & \\
Ar & 22,16 & 5,54 & 6,90 & & & \\
\hline
\end{tabular}

Tabla 8. Análisis de varianza con $\alpha=0,05$ sin subsoleo.

\begin{tabular}{ccccccc}
\hline Tratamiento & Suma & Promedio & Varianza & F & Probabilidad & Valor crítico F' \\
\hline Lc & 23,67 & 5,92 & 0,95 & 1,04 & 0,41 & 3,49 \\
Lo & 15,21 & 3,80 & 4,31 & & & \\
Rm & 18,49 & 4,62 & 1,73 & & & \\
Ar & 22,16 & 5,54 & 6,90 & & & \\
\hline
\end{tabular}

Tabla 9. Análisis de varianza con $\alpha=0,01$ con práctica de subsoleo.

\begin{tabular}{ccccccc}
\hline Tratamiento & Suma & Promedio & Varianza & F & Probabilidad & Valor crítico F' \\
\hline Lc & 24,06 & 6,02 & 1,66 & 0,69 & 0,57 & 5,95 \\
Lo & 17,26 & 4,32 & 4,44 & & & \\
Rm & 19,10 & 4,78 & 2,03 & & & \\
Ar & 22,23 & 5,56 & 5,31 & & & \\
\hline
\end{tabular}

Tabla 10. Análisis de varianza con $\alpha=0,05$ con práctica de subsoleo.

\begin{tabular}{ccccccc}
\hline Grupos & Suma & Promedio & Varianza & F & Probabilidad & Valor crítico para F \\
\hline Lc & 24,06 & 6,02 & 1,66 & 0,69 & 0,57 & 3,49 \\
Lo & 17,26 & 4,32 & 4,44 & & & \\
Rm & 19,10 & 4,78 & 2,03 & & & \\
Ar & 22,23 & 5,56 & 5,31 & & & \\
\hline
\end{tabular}

Basado en el nivel de significancia se determina que los tratamientos que ofrecen mayores rendimientos son labranza de conservación (Lc), Labranza con arado de discos (Ar) y labranza con rastra $(\mathrm{Rm})$, las que ofrecen mayores expectativas de desarrollo en regiones con lluvias escasas.

\section{Componentes de rendimiento}

Basado en el análisis de componentes de rendimiento y de acuerdo con los resultados obtenidos (Tabla 11) se determina que la densidad de población en la Tinaja presentó poca variación entre tratamientos (N.S), lo que sugiere que no existen problemas de variabilidad por efecto de la densidad de plantas por hectárea, germinación (calidad de la semilla, inadecuada cama de la semilla, siembra superficial e insuficiente humedad, entre otros) o pérdida de plantas durante el desarrollo del cultivo (por plagas o estrés).

La relación mazorca/planta $(\mathrm{Mz} / \mathrm{Pl})$ en el tratamiento de Lc registró la mayor relación, en $\mathrm{Rm}$ y Ar se registraron mazorcas en menos de la mitad de las plantas; en Lo esta relación fue negativa registrándose alto porcentaje de plantas sin producción de mazorca, traduciéndose como pérdida total por planta en el rendimiento de grano. Esta respuesta fue debida a efecto de factores limitantes como baja polinización y/o estrés del número de grano por mazorca $(\mathrm{Ng} / \mathrm{Mz})$; estos problemas registrados 
Tabla 11. Componentes de rendimiento de las localidades para los tratamientos en estudio.

\begin{tabular}{|c|c|c|c|c|c|c|}
\hline \multirow{2}{*}{ Rendimiento } & \multicolumn{4}{|c|}{ Tratamientos } & \multirow{2}{*}{ Factor B\%* } & \multirow{2}{*}{ Clasificación } \\
\hline & $\mathrm{Lc}$ & Lo & $\mathrm{Rm}$ & $\mathrm{Ar}$ & & \\
\hline \multicolumn{7}{|c|}{ La Tinaja } \\
\hline $\mathrm{Ng} \quad \mathrm{Mz} / \mathrm{Pl}$ & 0,58 & 0,13 & 0,41 & 0,38 & $\mathrm{AS}<1 \%$ & $\mathrm{Lc}>\mathrm{Rm}-\mathrm{Ar}>\mathrm{Lo}$ \\
\hline Pl $\quad \mathrm{Gr} / \mathrm{Mz}$ & 331 & 209 & 266 & 280 & $\mathrm{AS}<1 \%$ & $\mathrm{Lc}>\mathrm{Rm}-\mathrm{Ar}>\mathrm{Lo}$ \\
\hline \multicolumn{7}{|l|}{ P 1 G (gramos) } \\
\hline \multicolumn{7}{|c|}{ La Croix } \\
\hline $\mathrm{Ng} \quad \mathrm{Mz} / \mathrm{Pl}$ & 0,78 & 0,55 & 0,60 & 0,66 & $\mathrm{AS}<1 \%$ & $\mathrm{Lc}>\mathrm{Rm}-\mathrm{Ar}-\mathrm{Lo}$ \\
\hline $\mathrm{Pl} \quad \mathrm{Gr} / \mathrm{Mz}$ & 436 & 322 & 360 & 380 & $\mathrm{AS}<1 \%$ & $\mathrm{Lc}>\mathrm{Rm}-\mathrm{Ar}-\mathrm{Lo}$ \\
\hline P 1 G (gramos) & 486 & 419 & 425 & 428 & $\mathrm{~S} \mathrm{al} \mathrm{3,89 \%}$ & $\mathrm{Lc}>\mathrm{Rm}-\mathrm{Ar}-\mathrm{Lo}$ \\
\hline \multicolumn{7}{|c|}{ San Isidro } \\
\hline $\mathrm{Mz} / \mathrm{Pl}$ & 0,71 & 0,76 & 0,75 & 0,81 & NS & $\mathrm{Ar}-\mathrm{Lo}-\mathrm{Rm}-\mathrm{Lc}$ \\
\hline $\mathrm{Gr} / \mathrm{Mz}$ & 399 & 379 & 391 & 444 & $\mathrm{~S}$ al $7,11 \%$ & $\mathrm{Ar}>\mathrm{Lc}-\mathrm{Rm}-\mathrm{Lo}$ \\
\hline P 1 G (gramos) & 503 & 511 & 499 & 514 & NS & $\mathrm{Ar}-\mathrm{Lo}-\mathrm{Lc}-\mathrm{Rm}$ \\
\hline \multicolumn{7}{|c|}{ Alista } \\
\hline $\mathrm{Mz} / \mathrm{Pl}$ & 0,65 & 0,69 & 0,66 & 0,78 & NS & $\mathrm{Ar}-\mathrm{Lo}-\mathrm{Rm}-\mathrm{Lc}$ \\
\hline $\mathrm{Gr} / \mathrm{Mz}$ & 344 & 348 & 352 & 396 & $\mathrm{~S}$ al $10,85 \%$ & $\mathrm{Ar}>\mathrm{Rm}-\mathrm{Lo}-\mathrm{Lo}$ \\
\hline P 1 G (gramos) & 520 & 503 & 518 & 545 & NS & $\mathrm{Ar}-\mathrm{Lc}-\mathrm{Rm}-\mathrm{Lo}$ \\
\hline
\end{tabular}

$\mathrm{Ng} / \mathrm{Pl}=$ Número de granos por planta; $\mathrm{Gr} / \mathrm{Mz}=$ Granos por mazorca; $\mathrm{Mz} / \mathrm{Pl}=$ Mazorca por planta .

en el rendimiento se considera son presentados principalmente en la etapa de desarrollo floral, como efecto de la insuficiente acumulación de biomasa por jilote en desarrollo, al estrés ambiental (presencia de sequías), además de la alta densidad que normalmente reducen el número de mazorcas por planta. Esta respuesta en las localidades de la Croix y San Isidro en las relaciones Mz/Pl y Ng/Mz, fue semejante a la localidad de La Tinaja, donde en la Lo presentó los rendimientos más bajos, no así para la localidad de Alista donde el rendimiento más bajo se presentó en Lc.

Basados en el análisis de varianza, las relaciones de Mz/Pl y Ng/Mz para La Tinaja y La Croix, se identifica que los tratamientos considerados son altamente significativos $(\alpha=0,01)$, lo que significa que la variabilidad por factores limitantes (estrés ambiental, siembra superficial y baja polinización), presentan repercusión sobre estas relaciones; por el contrario, en las localidades San Isidro y Alista estas relaciones no fueron significativas, por lo tanto la variabilidad es baja entre relaciones, asumiendo entonces que no existió problemas de estrés ambiental, polinización baja, densidad alta o factores de manejo del suelo.

La dispersión y variabilidad de Mz/Pl y Gr/ $\mathrm{Mz}$ con respecto al rendimiento (Figura 3), registra una relación positiva; la mayor asociación de Mz/ $\mathrm{Pl}$ respecto al rendimiento (valores cercanos a 1 y variabilidad aproximada a 100\%) fue para La Croix y La Tinaja; con respecto a la relación $\mathrm{Ng} / \mathrm{Mz}$ y rendimiento, los cuatro sitios presentan asociación elevada y variabilidad cercana a $100 \%$.

Las relaciones de $\mathrm{Mz} / \mathrm{Pl}$ y $\mathrm{Ng} / \mathrm{Mz}$ respecto al rendimiento para los tratamientos establecidos, se observa una asociación importante (valores cercanos a 1) y una variabilidad cercana a $100 \%$ (Figura 4), siendo la máxima correlación para Lo; ello hace suponer que el aumento del número de mazorcas por planta aumentará el rendimiento del cultivo al obtener un rendimiento alto el número de mazorcas por planta será significativo.

La relación manifiesta entre el número de granos por planta y rendimiento se considera que es fuerte la asociación para Lo, no así para los demás tratamientos que presentan variabilidad aproximada a $65 \%$. Esta respuesta hace suponer que la cantidad de $\mathrm{Ng} / \mathrm{Mz}$ aumenta el rendimiento pero no directamente como se establece en el número de mazorcas por planta, excepto para Lo.

Finalmente se midieron las alturas y diámetro de plantas para cada tratamiento a las cinco semanas y en floración (Tabla 12), resultando significativas $(\alpha=0,01)$ para La Tinaja. Para La Croix y Alista 

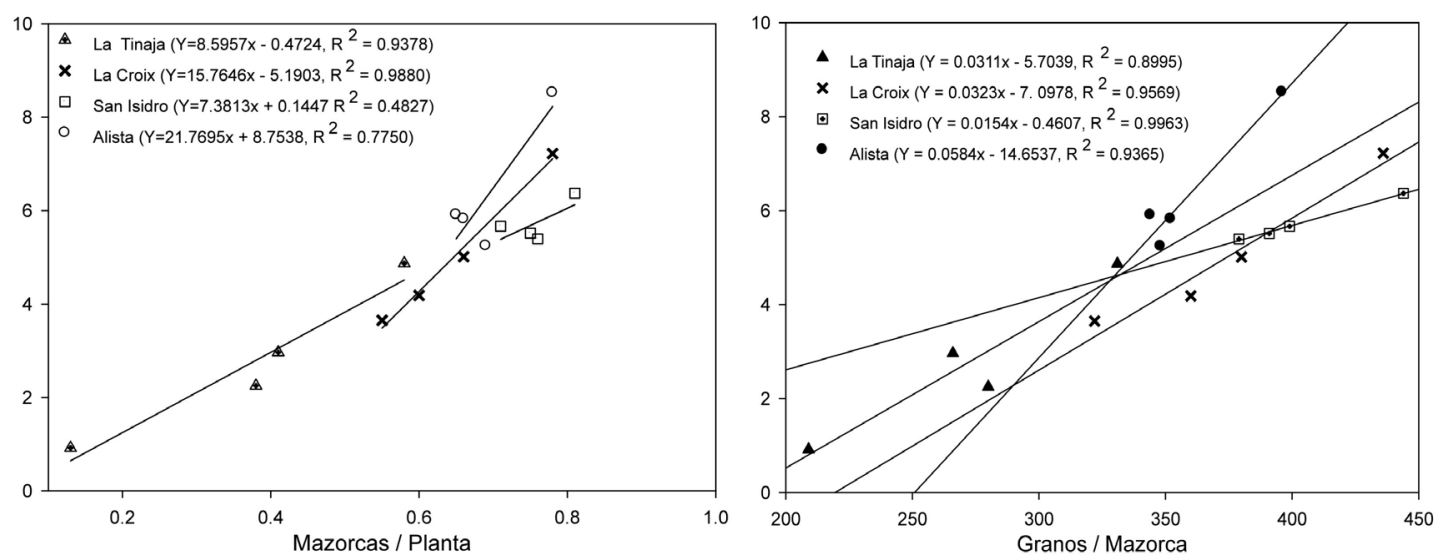

Figura 3. Análisis de regresión de Mz/Pl y Ng/Mz en relación al rendimiento.
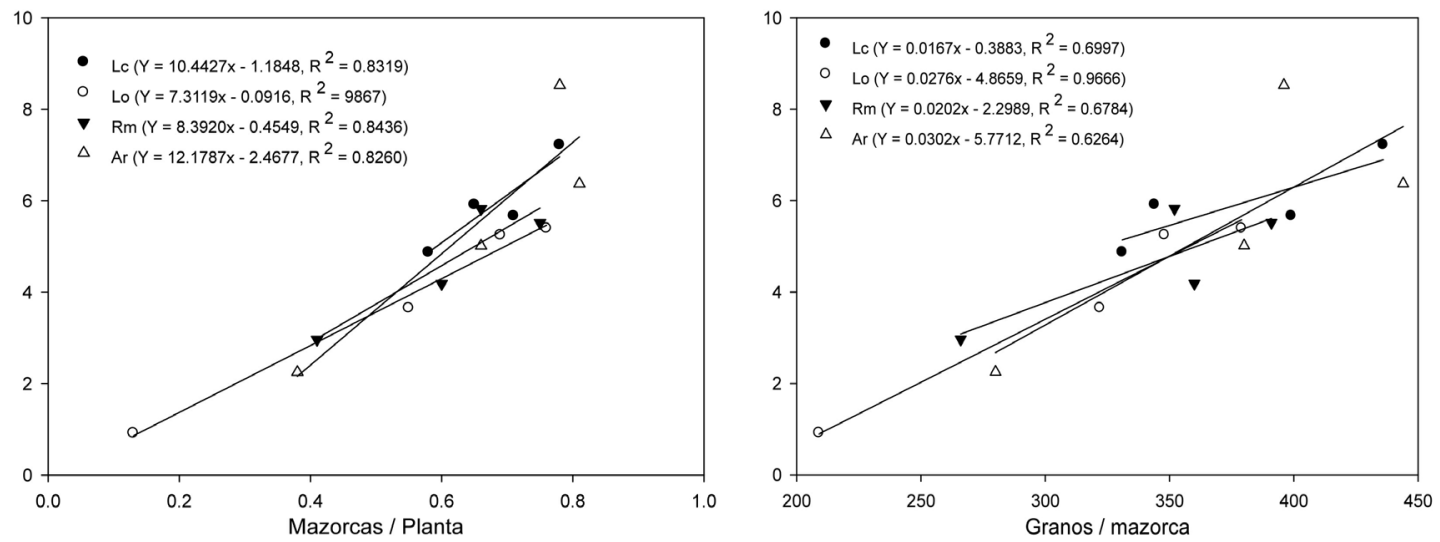

Figura 4. Análisis de regresión de Mz/Pl y Ng/Mz en relación al rendimiento.

Tabla 12. Descripción de mediciones en alturas y diámetro al cuadrado para los tratamientos.

\begin{tabular}{|c|c|c|c|c|c|c|}
\hline \multirow{2}{*}{ Alturas y diámetro } & \multicolumn{4}{|c|}{ Tratamientos } & \multirow{2}{*}{ Factor B\%* } & \multirow{2}{*}{ Clasificación } \\
\hline & $\mathrm{Lc}$ & Lo & $\mathrm{Rm}$ & $\mathrm{Ar}$ & & \\
\hline \multicolumn{7}{|c|}{ La Tinaja } \\
\hline A las 5 semanas & 894 & 704 & 836 & 878 & AS $1 \%$ & $\mathrm{Lc}-\mathrm{Ar}-\mathrm{Am}>\mathrm{Lo}$ \\
\hline A floración & 1.546 & 1.161 & 1.447 & 1.436 & $\mathrm{AS}<1 \%$ & $\mathrm{Lc}-\mathrm{Ar}-\mathrm{Am}>\mathrm{Lo}$ \\
\hline \multicolumn{7}{|c|}{ La Croix } \\
\hline A las 5 semanas & 800 & 778 & 770 & 786 & NS & $\mathrm{Lc}-\mathrm{Ar}-\mathrm{Lo}-\mathrm{Rm}$ \\
\hline A floración & 1.582 & 1.292 & 1.472 & 1.452 & NS & $\mathrm{Lc}-\mathrm{Rm}-\mathrm{Ar}-\mathrm{Lo}$ \\
\hline \multicolumn{7}{|c|}{ San Isidro } \\
\hline A las 5 semanas & 415 & 463 & 491 & 526 & S 5\% & $\mathrm{Ar}>\mathrm{Rm}>\mathrm{Lo}>\mathrm{Lc}$ \\
\hline A floración & 1.711 & 1.635 & 1.686 & 1.752 & NS & $\mathrm{Ar}-\mathrm{Lc}-\mathrm{Rm}-\mathrm{Lo}$ \\
\hline \multicolumn{7}{|c|}{ Alista } \\
\hline A las 5 semanas & 554 & 532 & 606 & 526 & NS & $\mathrm{Rm}-\mathrm{Lc}-\mathrm{Lo}-\mathrm{Ar}$ \\
\hline A floración & 1.531 & 1.410 & 1.400 & 1.435 & NS & $\mathrm{Lc}-\mathrm{Ar}-\mathrm{Rm}-\mathrm{Lo}$ \\
\hline
\end{tabular}


estas mediciones a las cinco semanas y en floración fueron no significativos $(\alpha=0,05)$; respuesta que fue similar en San Isidro únicamente en floración.

\section{Conclusiones}

Ante la presencia de períodos secos antes de la floración y posterior a esta, la labranza de conservación presentó mejor comportamiento al tener menor estrés hídrico así como limitantes de producción, lo que se manifiesta en mejor sincronización de la floración, mayor número de mazorcas por planta y granos por mazorca $\mathrm{y}$, en ciertas ocasiones, mayor peso de 1.000 granos para los sitios de la Tinaja y La Croix, esto como resultado de una fecundación más eficiente; lo anterior posiblemente debido a la mezcla de la materia orgánica y aumento en la humedad. Basado en Báez y Aguirre (2011) esta respuesta genera mejor estructura del suelo en textura franco-arenosa.

En los sitios de San Isidro y Alista, la labranza con discos presentó las mejores promedios y condiciones en nutrición mineral, volumen de suelo colonizado y aireación de las raíces, aunado a un mejor rendimiento por el aumento del espacio poroso en el suelo franco-arcilloso. Respuesta que Paustian et al. (1997), Ureste y Campos (1995), manifiestan es basada en que el subsoleo modifica la estructura al aumentar la aireación y la absorción de los minerales por las plantas.
En consideración a los resultados obtenidos se concluye que la labranza de conservación es efectiva en el incremento de rendimientos en suelos arenosos en comparación con los otros sistemas de labranza implementados. En suelos arcillosos, en respuesta al rendimiento la labranza con arado de disco fue el más efectivo favorecer la porosidad lo que ayuda la mejor absorción de los nutrientes.

Del análisis de varianza la respuesta del rendimiento entre sitios $(\alpha=0,05)$ con y $\sin$ subsoleo, se manifiesta una fuerte relación entre los valores de rendimiento con respecto a los factores climáticos y prácticas del suelo. En cuanto al análisis de varianza con $\alpha=0,01$ y $\alpha=0,05$ en función del rendimiento por tratamiento, se determina que la labranza de conservación, no necesariamente presenta los mejores resultados y perspectivas de desarrollo agrícola en relación a los otros tipos de labranza.

La comparación entre componentes de rendimiento, los máximos valores de mazorcas por planta, granos por mazorca y peso de 1.000 granos, fueron para los tratamientos de labranza de conservación y labranza con discos; valores bajos fueron para labranza cero y labranza con rastra, respuesta que sugiere que el estrés climático y prácticas de manejo influyeron sobre estos tratamientos.

Finalmente para las mediciones de alturas y diámetro, la mejor respuesta se presentó en la labranza de conservación bajo el supuesto que ante el estrés hídrico tuvo mejor respuesta.

\section{Literatura Citada}

Badii, M.H.; Castillo, J.; Rodríguez, M.; Wong, A.; Villalpando, P. 2007. Diseños experimentales e Investigación Científica. Innovaciones de Negocios, 4 (2): 283-330.

Báez, M.A.; Aguirre M.J.F.

2011. Efecto de la Labranza de Conservación sobre las propiedades del suelo. Terra Latinoamericana, 29 (2): 113-121.

Bauer, A.; Black, A.L.

1994. Quantification of the effect of soil organic matter content on soil productivity. Soil SciSoc. Am. J., 58: 185-193.

Bergamaschi, H.; Dalmago, G.A.; Comiran, F.; Bergonci, J.I.;

Muller, A.; Franca, S.; Santos, A.; Radin Biachi, C.B.; Pereira, P. 2006. Déficit hídrico y productividad en la cultura do milho. Pesq. Agropec. Bras., 41 (2): 243-249.

Broa, R.E.; Bahena, D.G.; Villarreal, M.L.A.; Valadez, R.M.; Jaime, H.M.A.

2013. Uso eficiente del agua en la producción de maíces nativos de color en Xalostoc, Morelos, México. Ambiente y Desarrollo, 17 (33): 99-110.
Buckles, D.; Barreto, H.J.

1996 Intensificación de sistemas de agricultura tropical mediante leguminosas de cobertura: un marco conceptual. CIMMYT, NRG 96-06, 13 p.

Cao, C.Y.; Jiang, S.Y.; Ying, Z.; Zhang, F.X.; Han, X.S.

2011. Spatial variability of soil nutrients and microbiological properties after the establishment of leguminous shrub Caraganamicrophylla Lam. plantation on sand dune in the Horqin Sandy Land of Northeast China. EcolEng., 37: 1467-1475.

Díaz, V.T.; Partidas, R.L.; Suárez, F.Y.E., Lizárraga, J.R.; López, L.A.

2014. Uso eficiente del agua y producción óptima en maíz con el uso de cuatro dosis de nitrógeno. Revista Ciencias Técnicas Agropecuarias, 23 (1): 32-36.

Eghball, B.; Ginting, D.; Gilley, J.E.

2004. Residual effects of manure and compost applications on corn production and soil properties. Agron. J., 96: 442-447. 
Gutiérrez, R.F.; Laguna, C.A.; Serrato, C,R.; y Valencia, B.I. 2006. Respuesta de cuatro variedades de maíz (Zea mays $L$.) en tres densidades de población y cuatro métodos de labranza. Revista Centro Agrícola, 33 (3): 9-15.

Galeana de la C.M.; Santos, A.T.; García, C., N.E.; Flores R.D. 2000. Labranza de Conservación y Fertilización en el Rendimiento de maíz y su efecto en el suelo. Terra Latinoamericana, 17 (4): 325-335.

García, H.C.; Rodríguez, M.G.D.

2012. Problemática y riesgo ambiental por el uso de plaguicidas en Sinaloa. Revista, Sociedad, Cultura y Desarrollo Sustentable, 8 (3): 1-10.

Instituto Nacional de Estadística y Geografía (México). 2013. Anuario estadístico y geográfico por entidad federativa 2013 / Instituto Nacional de Estadística y Geografía. México: INEGI, $744 \mathrm{p}$.

OCDE/FAO.

2013. OCDE-FAO Perspectivas Agrícolas 2013-2022. Universidad Autónoma Chapingo. Texcoco, México, 335 p.
Ortiz-Torres, E.; López, P.A.; Gil-Muñoz, A.; GuerreroRodríguez, J. de D.; López-Sánchez, H.; Taboada-Gaytán, O.; Hernández-Guzmán, J.A.; Valadez-Ramírez, M.

2013. Rendimiento y calidad de elote en poblaciones nativas de maíz de Tehuacán, Puebla. Revista Chapingo. Serie horticultura, 19 (2): 225-238.

Paustian, K.; Andrén, O.; Janzen, H.H.; Lal, R.; Smith, P.; Tian, G.; Tiessen, H.; Van Noordwijk, M.; Woomer, P.L. 1997. Agricultural soils as a sink to mitigate $\mathrm{CO}_{2}$ emissions. Soil Use Management, 13 (4): 230-244.

Ureste, J.; Campos, S.

1995. Respuesta del cultivo de maíz a la labranza de conservación en cuatro diferentes suelos tropicales. Agronomía Mesoamericana, 6: 80-87.

Wang, T.; Hu, J.; \& Liu, C.

2014. Simultaneous determination of insecticide fipronil and its metabolites in maize and soil by gas chromatography with electron capture detection. Environmental Monitoring and Assessment, 186 (5), 2767-2774. 
